\title{
Three-Dimensional Reconstruction of Actin in a Sensory Glial Cell Using Bi-plane PALM
}

Shigeki Watanabe*, Carl Ebeling**, Grigorios Oikonomou***, Shai Shaham***, Jordan M. Gerton** and Erik M. Jorgensen*

* Howard Hughes Medical Institute and Department of Biology, University of Utah, 257 South 1400 East Salt Lake City, UT 84112

** Department of Physics, University of Utah, 115 South 1400 East, Salt Lake City, UT 84112 *** Laboratory of Developmental Genetics, the Rockefeller University, 1230 York Avenue, New York, NY 10065

To fully understand the function of proteins, the location of proteins within a cell must be defined. Since the discovery of green fluorescent protein, the subcellular localization of proteins using fluorescence microscopy has become possible. However, fluorescence images are limited in resolution; although GFP is only $2.5 \mathrm{~nm}$ in diameter, the fluorescence signal appears as a 400nm spot because of the diffraction limit of light [1]. Recently, this limitation has been overcome by several microscopy techniques [2]. Photo-activated localization microscopy (PALM) [3] and related techniques (stochastic optical reconstruction microscopy, STORM [4]; fluorescence photoactivation localization microscopy, fPALM [5]) improve resolution by limiting the number of excited molecules in a broad field. By stochastically activating only individual molecules separated by a 200 $\mathrm{nm}$ radius at a given time, the emission pattern from each fluorophore is recorded, and the centroid of each is subsequently calculated. The locations of the individual centroids of each molecule are then mapped on the final image. However, PALM methods do not portray three-dimensional structure.

PALM often employs evanescent field illumination -the excitation light is projected onto the coverslip at a high angle, resulting in total internal reflection; the resultant evanescent wave that propagates into the sample can be used to stimulate fluorescent molecules within $100 \mathrm{~nm}$ of the surface of the coverslip. The resulting image from this configuration only represents molecules in two-dimensions, and the distribution of proteins deeper in the cell cannot be mapped using this approach. Bi-plane PALM [6] and several other techniques [7-9] have been developed to overcome this limitation.

In the bi-plane system, the fluorescence signal collected by the objective lens is split into two beams via a 50/50 beam splitter. The transmitted light propagates straight to the camera and is imaged on one half of the CCD chip while the reflected light takes a longer light path and is imaged on the other half of the CCD chip. The extended light path for the reflected light shifts the focal plane at the specimen; therefore, two focal planes are simultaneously imaged onto the camera. The distribution of light in each focal plane can be fit to a plane orthogonal to the propagation axis of a three-dimensional point spread function. These planes are then used to calculate the precise location of the signal in three-dimensions. Thus bi-plane PALM allows three-dimensional mapping of fluorescent molecules at super-resolution.

In theory, three-dimensional protein localization at a single-molecule level is possible using bi-plane PALM. However, the application has so far been limited to moderately flat specimens such as cultured cells [6]. Here, we imaged actin molecules in the amphid sheath glia cells from the intact Caenorhabditis elegans animal using bi-plane PALM. The amphid sensory neurons and glia cells are beneath the cuticle and hypodermal cells near the nose of the worm. The glial sheath cell surrounds the ciliated endings of the sensory neurons to compartmentalize the sensory endings. The channels in 
the glial cell where the cilia pass are narrow, and thus fluorescence from tagged actin molecules in the glia cell appears as a blob using conventional confocal microscopy (FIG. 1). Bi-plane PALM revealed that the blob contains a hollow tube in the middle, presumably where cilia bundles pass through (FIG. 2). These results demonstrated that the three-dimensional distribution of proteins tagged with genetically encoded fluorophores can be revealed even from a thick sample $(\sim 30 \mu \mathrm{m})$.

\section{References}

1. Cox, G. \& Sheppard, C.J. Practical limits of resolution in confocal and non-linear microscopy. Microscopy Research and Technique 63, 18-22 (2004).

2. Hell, S.W. Far-field optical nanoscopy. Science 316, 1153-1158 (2007).

3. Betzig, E. et al. Imaging Intracellular Fluorescent Proteins at Nanometer Resolution. Science 313, 1642-1645 (2006).

4. Rust, M.J., Bates, M. \& Zhuang, X. Sub-diffraction-limit imaging by stochastic optical reconstruction microscopy (STORM). Nat. Methods 3, 793-795 (2006).

5. Hess, S.T., Girirajan, T.P.K. \& Mason, M.D. Ultra-high resolution imaging by fluorescence photoactivation localization microscopy. Biophys. J 91, 4258-4272 (2006).

6. Juette, M.F. et al. Three-dimensional sub-100 $\mathrm{nm}$ resolution fluorescence microscopy of thick samples. Nat. Methods 5, 527-529 (2008).

7. Huang, B., Wang, W., Bates, M. \& Zhuang, X. Three-dimensional super-resolution imaging by stochastic optical reconstruction microscopy. Science 319, 810-813 (2008).

8. von Middendorff, C., Egner, A., Geisler, C., Hell, S.W. \& Schönle, A. Isotropic 3D Nanoscopy based on single emitter switching. Opt Express 16, 20774-20788 (2008).

9. Shtengel, G. et al. Interferometric fluorescent super-resolution microscopy resolves 3D cellular ultrastructure. Proceedings of the National Academy of Sciences 106, 3125-3130 (2009).

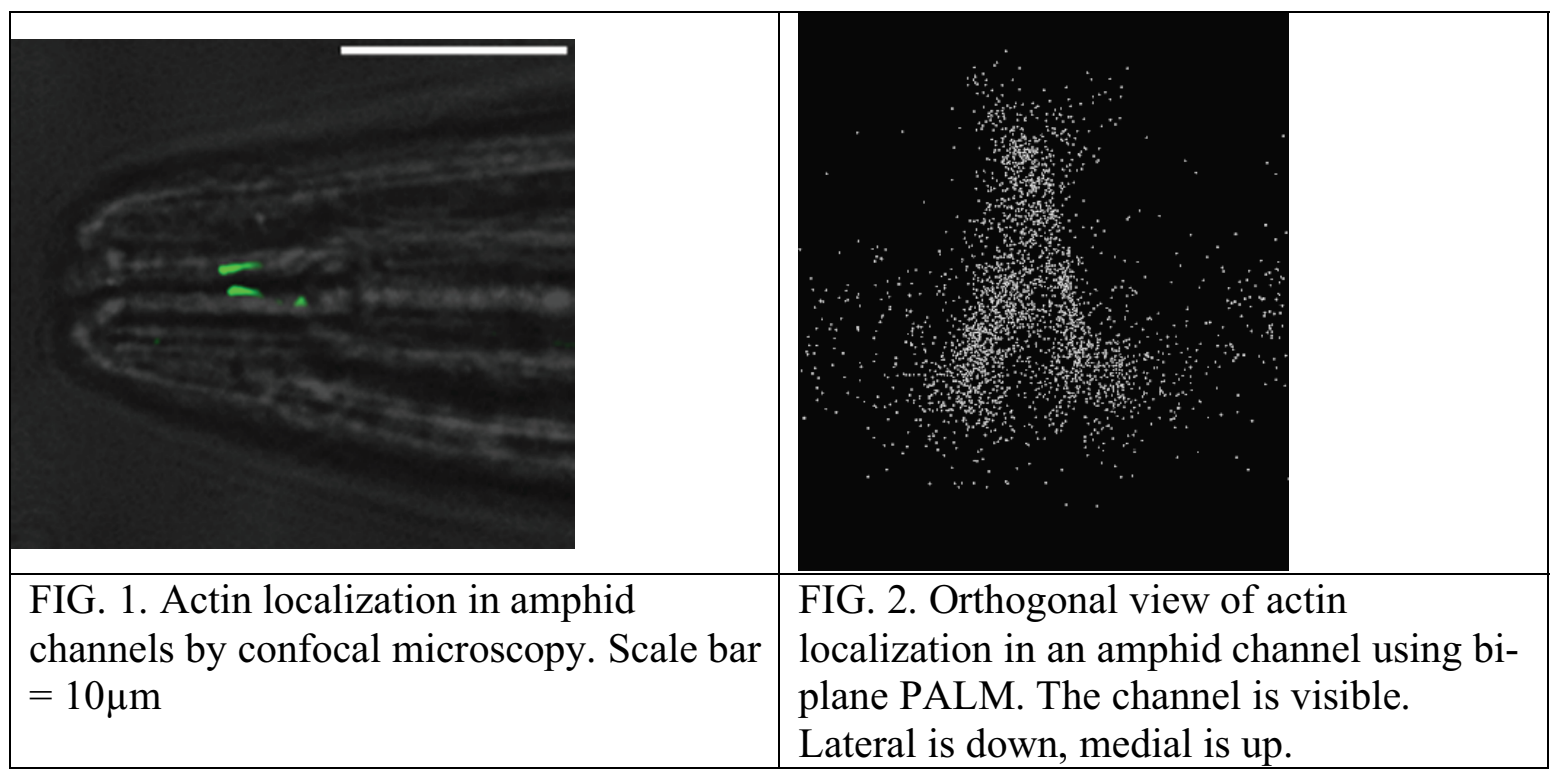

